\title{
Schizophrenia: An overview
}

Schizophrenia is a serious mental disorder that affects how a person thinks, feels, and behaves. People with schizophrenia may seem like they have lost touch with reality. They may hear voices other people don't hear. Schizophrenia symptoms contain three categories; Positive, Negative and Cognitive. There are different causes of Schizophrenia this may be due to Genes, Environment or Change in Brain Structures. Due to Schizophrenia the illness occurs in less than 1 percent of the general population, but this range becomes 10 percent who have first degree relatives with the disorder, such as parents, Brother or sister. Most people with schizophrenia are not violent; however, the risk of violence is greatest when schizophrenia is untreated. There are different molecular mechanisms of Schizophrenia i.e. Neurodevelopment Hypothesis, Dopamine hypothesis and Glutamate Hypothesis. It is important to help a person with schizophrenia symptoms get treatment as quickly as possible. Here Two main types of treatment can help with symptoms: antipsychotic medications and psychosocial treatments. Family and friends can help their loved ones with schizophrenia by helping them get treatment and encouraging them to stay in treatment.

\section{Keywords: schizophrenia, hallucination, glutamate hypothesis.}

\section{Introduction}

Schizophrenia is a chronic psychiatric disorder with a heterogeneous genetic and neurobiological background that influences early brain development and is expressed as a combination of psychotic symptoms such as hallucinations, delusions and disorganization and motivational and cognitive dysfunctions. Although Schizophrenia affects men and women with equal frequency. Schizophrenia is not as common as other mental diseases it can be very disabling as approximately 7-8 individuals out of 1000 will have this disorder. Schizophrenia is a word used to describe a mental disorder which has a spectrum of symptoms including alterations in perception, thought and sense of a self-decrease in violation, psychomotor slowing and displays of antisocial behaviour [1].

\section{- Clinical characteristics}

Schizophrenia has varied symptoms that generally begin in early adulthood and usually continue throughout life. Most patients have a history of behavioural dysfunction primarily social and learning difficulties. Diagnostic features of schizophrenia include auditory hallucinations (an experience involving the apparent perception of something not present) and delusions (the action of deluding or the state of being deluded). Patients may have experienced these symptoms but this phenomenon may or may not true and now in its troubling condition. Schizophrenia has different main symptoms which can be divided into different phases which are; Positive, Negative and Cognitive symptoms. Positive symptoms are those which can be easily identified and not seen in healthy people. Such symptoms include Hallucination, delusion and abnormal motor behaviour having fluctuating degree of severities. Negative symptoms are can't easily identify and associated with high morbidity rate. The most common Negative symptoms included Avoilition, Alogia, Anhedonia and diminished emotional expression. Cognitive Symptoms, being the newest classification. These ultimately impairing the individual's communicating skills by disturbing his speech and attention $[2,3]$.

\section{- Aetiology}

Research has identified several factors that contribute to the risk of developing

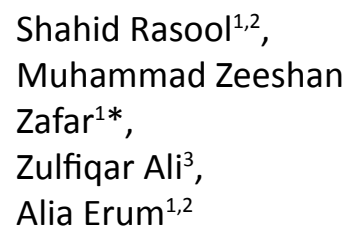

Shahid Rasool ${ }^{1,2}$, Muhammad Zeeshan Zafar $^{1 *}$, Zulfiqar Ali ${ }^{3}$, Alia Erum ${ }^{1,2}$

${ }^{1}$ Department of Pharmacy, University of Sargodha, Pakistan

${ }^{2}$ Department of Pharmacognosy, University of Sargodha, Pakistan

${ }^{3}$ Department of Paeeds, Gujranwala Medical Colleg, Pakistan

*Author for correspondence:

shanmughal11@gmail.com 
schizophrenia. Scientists have long known that schizophrenia sometimes runs in families. The illness occurs in less than 1 percent of the general population, but this range becomes 10 percent who have first degree relatives with the disorder, such as parents, Brother or sister. Many environmental factors may be involved, such as exposure to viruses or malnutrition before birth, problems during birth, and other not yet known, psychosocial factors (Schizophrenia). Scientists also believe that brain structure of the people with schizophrenia is slightly different than healthy peoples. For example, fluid-filled cavities at the center of the brain called ventricles are larger in some people with schizophrenia. Other most common cause of schizophrenia evidenced that most people identified with schizophrenia have increased in dopamine level but it's still not known that how everyone diagnosed with schizophrenia have too much dopamine $[4,5]$.

\section{- Epidemiology}

Schizophrenia occurs throughout the world. The prevalence of schizophrenia approaches 1 percent internationally. The incidence is about 1.5 per 10000 people. Slightly more men are diagnosed with schizophrenia than women at an early age, but women are more common in later age. Typical age of onset in 18 to 25 for men and $25-35$ is for women. On the other hand, a more recent review, which included data from 33 countries, concluded that the incidence of schizophrenia varied by geographic location $[6,7]$.

\section{Molecular mechanisms of Schizo- phrenia}

There are different mechanisms related to schizophrenia given below;

\section{- Neurodevelopment hypothesis}

The neurodevelopment hypothesis of schizophrenia postulates that effects during an embryonic or fetal stage in brain development lead to defective neural activity and altered neuronal functioning later in life. The alterations observed in post mortem of a schizophrenic patient that neuro developmental disturbances mainly related to the hippocampal formation and in the superior temporal lobe. The neurodevelopmental abnormalities developing in utero as early as late first or early second trimester and which leads to in young adulthood appear the positive and negative symptoms or both [7-9].

\section{Dopamine hypothesis}

The most widely contemplated neuro chemical hypothesis of schizophrenia is the dopamine hypothesis, which theorizes that symptoms of schizophrenia may results from excess dopaminergic neurotransmission particularly in mesolimbic and striatal brain regions which lead to positive symptoms and finally changes into schizophrenia. There are many clinical shreds of evidence about schizophrenia that provides support for the dopamine hypothesis. In this hypothesis, the different evidence has appeared. The first evidence that in schizophrenia patient's dopamine came from amphetamine users. Amphetamine showed that too produces more dopamine and produces psychotic symptoms related to schizophrenia $[10,11]$.

\section{Glutamate hypothesis}

In this Hypothesis, it is noted that dopaminergic dysfunctioning may be associated with glutamatergic dysfunctioning. In this concept glutamate, dysfunctioning will lead to opening effect in the thalamocortical loop which causes to appear psychotic symptoms and well-known dopamine concentration changes. Glutamatergic receptors consist of two groups which can perform different functions and finally lead to schizophrenic symptoms appear. In these receptors, major receptors are NMDA (N-methyl, D-Aspartate) receptor which causes schizophrenia among most patients by changing dopamine level from the normal range $[13,14]$.

\section{Current treatment options for Schizophrenia}

Recently there are several treatment options used for to curing of schizophrenia and in practice. Mainly schizophrenia therapy is given by indicating this with clinical reports and their ranges or severity. Patients with schizophrenia who can't receive their medications may increase the risk of relapse, which can lead to hospitalization. Therefore it's important to inform the patient about his illness and current disease condition. As you know schizophrenia is a severe chronic illness that requires lifelong medication, therefore, its duty to maintain this. 
Main drug therapy used for schizophrenia is with antipsychotic agents [15].

\section{Antipsychotic agents for Schizo- phrenia}

First generation antipsychotics (FGAs) drug, Chlorpromazine which was discovered in 1950 and used commonly because at that time this only antipsychotic drug and used for the treatment of schizophrenia. Chlorpromazine's intensity will reduce the intensity of schizophrenia. Also in this class may other agents was also discovered by changing structure and activities which are loxapine, fluphenazine, perphenazine, and haloperidol, but these all agents have a major side effect, extrapyramidal symptoms and this can't be neglected. Therefore these drug no use longer. These FGAs drugs also called as typical or conventional drug [16].

Clozapine is another medication of second generation antipsychotic agents which was discovered in 1970 and is much better than other agents. It is dopamine receptor blocker but blocks in less extent. Second generation antipsychotics (SGAs) are used as a first line treatment for schizophrenia and effective for this treatment. After this number of SGAs was also discovered which can also have good efficiency for to reduce the severity of this disease? This class first called as atypical antipsychotic drugs, but now known as second generation antipsychotics, was heralded as the first major advance in the therapeutics of schizophrenia for 40 years. These drugs seem to have more advantages on first generation antipsychotics [17].

In most schizophrenia patients, it is difficult to implement effective rehabilitation programs without antipsychotic agents. In the event of the acute psychotic episode, drug therapy should be administered immediately.

Clozapine efficiency may increases if given in combination with other therapies like its efficiency enhances if given with risperidone. Clozapine is more effective than other antipsychotic agents.

Clozapine started given with monitoring of white blood cells (WBCs) count. By its heavy dose if agranulocytosis occurs then must discontinue the clozapine. If schizophrenia in a severe stage then must be combination therapies are useful and which may be a combination of SGAs with FGAs or other agents. Second generation antipsychotics (SGAs) have not any prominent side effects but the major side effect is to weight gain. Moreover, high doses clozapine has been associated with serious adverse effects, such as seizure. So these are some major antipsychotics which use commonly $[18,19]$.

\section{Augmentation and combination therapies}

Augmentation therapy is given with second generation antipsychotics combine with electroconvulsive therapy (ECT) or mood stabilizing agents on the other hand combination therapy is given with other antipsychotic agents.

Lithium is commonly used as mood stabilizing agent. Lithium may improve the mood and behavior but doesn't have any antipsychotic effect [16].

\section{Mechanisms common drugs used in Schizophrenia}

There are numerous drugs used for to treat schizophrenia and they have the different mechanism of actions for to produce an effect in the body [19]. Especially antipsychotics are used for Schizophrenia, therefore here mechanisms related to antipsychotics given below:

\section{- First generation antipsychotics}

First generation antipsychotics (FGAs) also known as, typical antidepressant, dopamine antipsychotics or Classic antipsychotics. The exact mechanism of action is unknown, But there mechanism of action is that these are phenothiazines derivatives and by binding with D2 receptors present in brain and act as antagonizers these also depresses the release of hypothalamic and hypophyseal harmones, also blocking mesolimbic dopamine receptors. So through these routes FGAs are blocking dopamine receptors. Positive symptoms are shown due to overactive of different pathways like a mesolimbic pathway, mesocortical pathway, and nigrostriatal pathway etc... When dopamine neurotransmitter mechanism activated then due to this over activity of dopamine these pathways are activated and produce positive symptoms of schizophrenia shown in FIGURE 1. But FGAs inhibiting the neurotransmitter dopamine receptor 2 activity due to which these pathways are can't activate and inhibited schizophrenia [20-22]. 


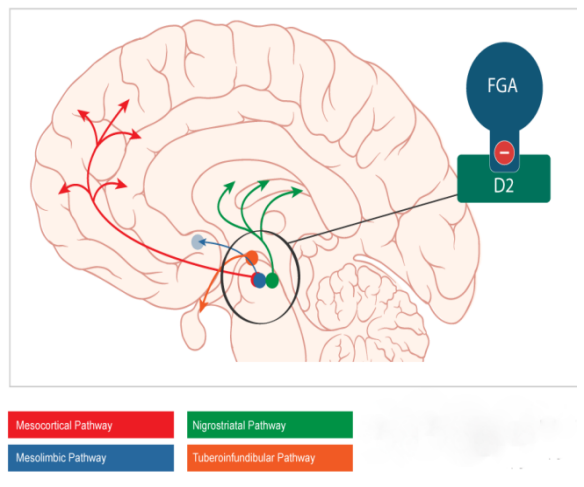

FIGURE 1. Mechanism Pathways for First generation Anti-psychotics (FGAs).

\section{- Second generation antipsychotics}

SGAs are also called as atypical antipsychotic agents. SGAs were discovered recently and improve schizophrenia treatment with fewer risks. Many medications used for schizophrenia are having higher risks with low efficiency. SGAs like clozapine is a strong serotonin antagonist, with strong binding to 5-HT, $2 \mathrm{~A} / 2 \mathrm{C}$ receptor subtypes. It also shows affinity with dopaminergic (D2) receptors but with weak extent. Clozapine show two types of mechanism of action in a combination form as antagonistic effect at D2 receptors in the mesolimbic pathway and 5-HT/2A receptor in the frontal cortex. As the blocking of D2 and 5-HT receptors, there is a reduction in the activation of mesolimbic pathways and frontal cortex respectively. D2 antagonism relieves positive symptoms, while $5-\mathrm{HT} / 2 \mathrm{~A}$ antagonist alleviates negative symptoms [23-25].

The use of these medications one major problem which faced is by repeated use of these agents of normal doses causes super-sensitivity in patients that not any further effect produced in a patient, therefore we need to increase the dose for to become cured or for producing an effect.

There are many drugs which induce dopamine level in body and cause of schizophrenia in patients, for example, Cocaine, Amphetamine etc. are those agents which improve dopamine level in the body.

\section{Conclusion}

We concluded from this study that Schizophrenia is a complex, chronic mental health disorder characterized by an array of symptoms, including delusions, hallucinations, disorganized speech or behavior. Schizophrenia is a complex disorder that requires prompt treatment. Although patients can increase adaptive functioning through available pharmacological and nonpharmacological treatment options, it is hoped that future research will address gaps in treatment and potentially a cure for schizophrenia.

\section{Author's Contribution}

MZZ and D.SR conceptualized the study and supervised experimental work; MZZ and $\mathrm{AE}$ extract and select appropriate data for paper from previous researches; D.ZA. wrote the paper and checked by MZZ.

\section{Conflicts of Interest}

The authors declare no conflict of interest. 


\section{REFERENCES}

Kahn RS, Sommer IE, Murray RM, et al. Schizophrenia. Nat. Rev. Dis. Primers. 15067 (2015).

Freedman R. Schizophrenia. N. Engl. J. Med. 349(18), 1738-1749 (2003).

http://www.schres-journal.com/ issue/S0920-9964(00)X0120-X

Weinberger DR, Levitt P. Neurodevelopmental Origins of Schizophrenia. Schizophrenia 393412 (2011).

Abel KM, Drake R, Goldstein JM. Sex differences in schizophrenia. Int. Rev. Psychiatry 22: 417 (2010).

Messias E, Chen CY, Eaton WW. Epidemiology of Schizophrenia: Review of Findings and Myths. The Psychiatr. Clin. North. Am. 30(3), 323-338 (2007).

McGrath J, Saha S, Welham J, et al. A systematic review of the incidence of schizophrenia: the distribution of rates and the influence of sex, urbanicity, migrant status, and methodology. BMC. Med. 2: 13 (2004).

Fatemi SH, Folsom TD. The Neurodevelopmental Hypothesis of Schizophrenia, Revisited. Schizophr. Bull. 35(3), 528-548 (2009).

Weinberger D. Cell biology of the hippocampal formation in schizophrenia. Biol. Psychiatry. 45(4), 395-402 (1999).
Coyle JT. NMDA Receptor and Schizophrenia: A Brief History. Schizophr. Bull. 38(5), 920926 (2012).

Gründer G, Cumming P. The Dopamine Hypothesis of Schizophrenia. The Neurobiology of Schizophrenia 109-124 (2016). Jentsch JD, Roth RH. The neuropsychopharmacology of phencyclidine: from NMDA receptor hypofunction to the dopamine hypothesis of schizophrenia. Neuropsychopharmacology 20(3), 201225 (1999).

Hu W, MacDonald ML, Elswick DE, Sweet RA. The glutamate hypothesis of schizophrenia: evidence from human brain tissue studies. Ann. N. Y. Acad. Sci. 1338(1), 38-57 (2015).

Clinton SM, Meador-Woodruff JH. Abnormalities of the NMDA Receptor and Associated Intracellular Molecules in the Thalamus in Schizophrenia and Bipolar Disorder. Neuropsychopharmacology. 29(7), 1353-1362 (2004).

Lewis S. Treatment of Schizophrenia: Discussion. J. Schizophr. Res. 2009, 441-445 (2009).

Patel KR, Cherian J, Gohil K, Atkinson D. Schizophrenia: Overview and Treatment Options. P. T. 39(9), 638-645 (2014).

Freedman R. The Choice of Antipsychotic Drugs for Schizophrenia. N. Engl. J. Med. 353(12), 1286-1288 (2005).

Davis JM. The Choice of Drugs for Schizophrenia. N. Engl. J. Med. 354(5), 518-520 (2006).

Harrison PJ. The neuropathological effects of antipsychotic drugs. Schizophr. Res. 40(2), 87-99 (1999).

Kane JM, Malhotra A. The future of pharmacotherapy for schizophrenia. World Psychiatry. 2(2), 81-86 (2003).

Harrigan P. New anti-psychotics in sight for schizophrenics. The Lancet 347(9018), 1822 (1996).

Ryall RW. Drugs used in schizophrenia. Mechanisms of Drug Action on the Nervous System 171192.

Meltzer HY. The Mechanism of Action of Novel Antipsychotic Drugs. Schizophr. Bulletin 17(2), 263-287 (1991).

Bishara D, Taylor D. Upcoming Agents for the Treatment of Schizophrenia. Drugs 68(16), 22692292 (2008).

Girgis RR, Javitch JA, Lieberman JA. Antipsychotic drug mechanisms: links between therapeutic effects, metabolic side effects and the insulin signaling pathway. Mol. Psychiatry 13(10), 918-929 (2008). 